\title{
A comparison of the effects of desflurane and propofol on the optic nerve sheath diameter during robot-assisted laparoscopic radical prostatectomy
}

\author{
Young-Tae Jeon, MD, PhD. Jihun JO, M.D., Seoul National University Bundang Hospital, \\ Dept of Anesthesiology \& Pain Medicine, Seongnam-si, Korea, Republic of
}

\begin{abstract}
Background: Use of the steep Trendelenburg position and high pressure $\mathrm{CO} 2$ pneumoperitoneum in patients undergoing robot-assisted laparoscopic radical prostatectomy (RALP) increase intracranial pressure (ICP). Volatile anesthetics including desflurane and propofol have different effects on ICP, cerebral blood flow. In this study, we measured sonographic Optic Nerve Sheath Diameters (ONSD) in patients undergoing RALP to evaluate intracranial pressure and compared the effects of desflurane and propofol.
\end{abstract}

\begin{abstract}
Materials and Methods: Sixty patients scheduled for RALP were randomly assigned to undergo desflurane with remifentanil (desflurane group) or propofol with remifentanil (TIVA group) anesthesia. We measured both ONSDs and recorded the average of values. Sonographic ONSDs and hemodynamic parameters were measured at specific time points: in the supine position after induction of anesthesia (T0), $10 \mathrm{~min}$ after the steep Trendelenburg position with pneumoperitoneum (T1), one hour after the steep Trendelenburg position combined with pneumoperitoneum (T2), two hour after the steep Trendelenburg position combined with pneumoperitoneum (T3), $10 \mathrm{~min}$ after in the supine position after desufflation of the pneumoperitoneum (T4) and 20 min after arrival in the post anesthetic care unit (T5).
\end{abstract}

Table 1. Patient Characteristics

\begin{tabular}{|l|l|l|l|}
\hline & \multicolumn{1}{|c|}{ Desflurane group } & \multicolumn{1}{|c|}{ TIVA group } & \\
\hline Age & $66.0 \pm 6.0$ & $66.4 \pm 8.2$ & 0.860 \\
\hline Height & $165.4 \pm 7.0$ & $166.2 \pm 4.5$ & 0.585 \\
\hline Weight & $66.8 \pm 6.1$ & $67.1 \pm 7.2$ & 0.895 \\
\hline BMI & $24.2 \pm 1.7$ & $24.4 \pm 2.5$ & 0.636 \\
\hline ASA & & & \\
\hline Total IV infused fluid & & & \\
\hline Operation time & $173.9 \pm 40.7$ & $173.3 \pm 27.2$ & 0.950 \\
\hline Anesthetic time & $217.6 \pm 43.1$ & $214.5 \pm 27.8$ & 0.744 \\
\hline DM & 24 & 26 & 0.731 \\
\hline HTN & 18 & 20 & 0.789 \\
\hline Lung disease & 28 & 28 & 1.000 \\
\hline
\end{tabular}

Table 2. Changes of MBP and ETCO2

\begin{tabular}{|l|l|l|l|}
\hline & $\begin{array}{c}\text { Desflurane group } \\
(\mathbf{n = 2 9})\end{array}$ & \multicolumn{1}{|c|}{$\begin{array}{c}\text { TIVA group } \\
(\mathbf{n = 3 0 )}\end{array}$} & \multicolumn{1}{|c|}{ P value } \\
\hline MBP $(\mathbf{m m H g})$ & & & \\
\hline T0 & $68.6 \pm 10.0$ & $65.0 \pm 6.0$ & 0.098 \\
\hline T1 & $95.0 \pm 18.0$ & $91.5 \pm 11.8$ & 0.376 \\
\hline T2 & $77.8 \pm 15.1$ & $75.4 \pm 13.4$ & 0.527 \\
\hline T3 & $70.8 \pm 22.2$ & $77.4 \pm 9.0$ & 0.135 \\
\hline T4 & $79.0 \pm 9.7$ & $81.3 \pm 11.9$ & 0.414 \\
\hline T5 & $87.9 \pm 13.2$ & $85.7 \pm 12.8$ & 0.512 \\
\hline ETCO2 $(\mathbf{m m H g})$ & & & \\
\hline T0 & $30.7 \pm 2.0$ & $30.2 \pm 1.6$ & 0.339 \\
\hline T1 & $31.7 \pm 3.2$ & $32.0 \pm 2.5$ & 0.613 \\
\hline T2 & $32.9 \pm 3.6$ & $32.8 \pm 2.2$ & 0.937 \\
\hline T3 & $30.2 \pm 10.9$ & $33.7 \pm 5.2$ & 0.057 \\
\hline T4 & $33.7 \pm 2.5$ & $33.8 \pm 3.4$ & 0.911 \\
\hline
\end{tabular}

Results and Discussion: Patient characteristics were similar between the two groups. Preoperative and intraoperative data associated with underling diseases, anesthesia time, operating time were not significantly different between the two groups (table 1). The MAP and ETCO2 at defined moments were comparable between two groups (table 2). The mean ONSD at time points (T1, T2, T3, T4, and T5) after anesthesia significantly increased compared with that before induction of anesthesia (T0). The change of ONSD at T1, T2, T3, T4 were significantly less in the TIVA group compared to the DES group (Fig 1). Neurological complications were not observed in any of the enrolled patients during the perioperative period of RALP.

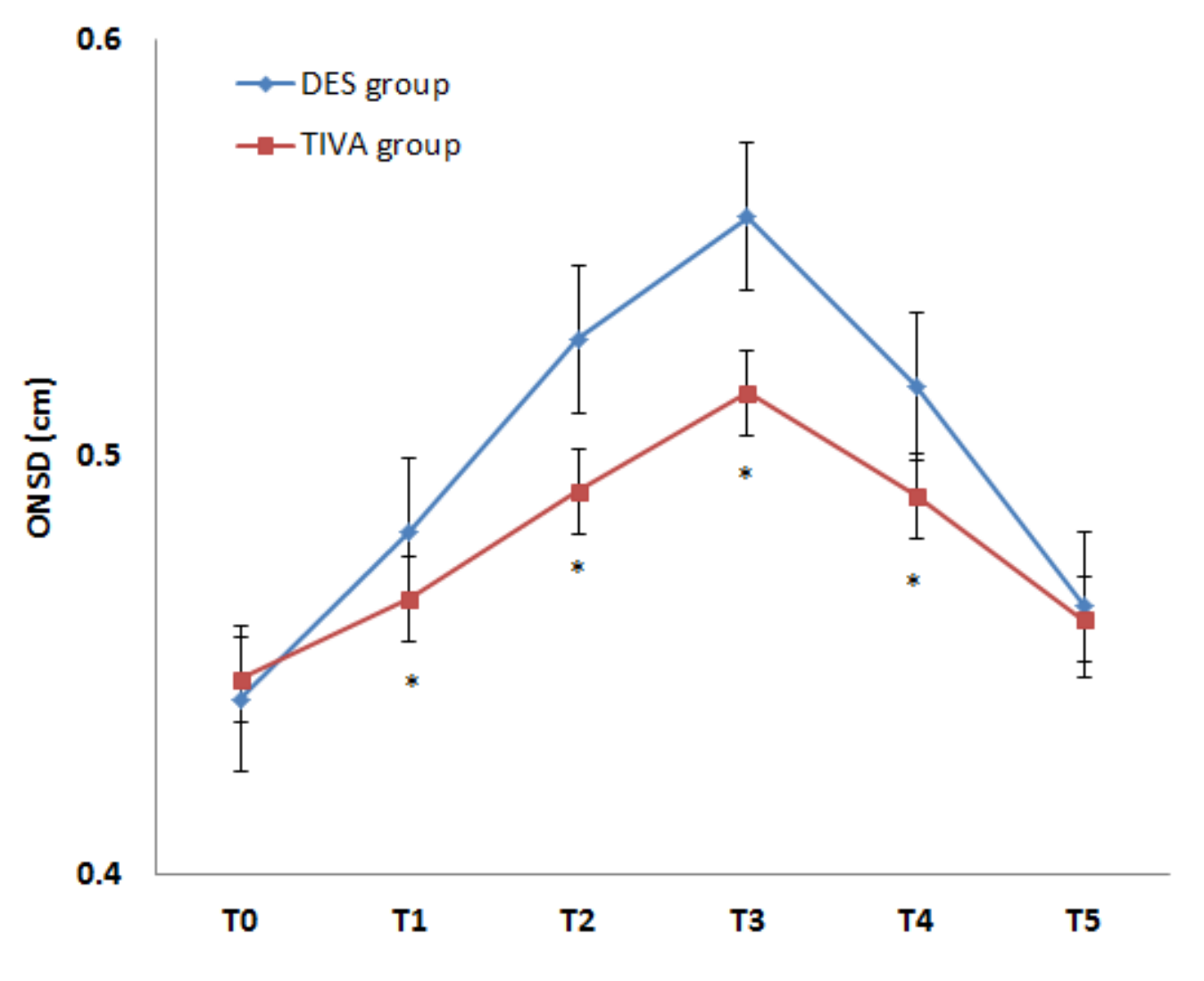

Fig. 1. Changes in optic nerve sheath diameter (ONSD) values between the two groups (TIVA group vs. desflurane group)

Conclusion(s): Trendelenburg position and carbon dioxide pneumoperitoneum in RALP increased the diameter of optic nerve sheath. The change of ONSD in TIVA group was significantly less than that in desflurane group during operation time in RALP. Through this results, TIVA could be a better choice for patients with the risk of cerebral hypoperfusion or with increased intracranial pressure.

References: Kim MS et al. J Endourol. 2014;28:801-6. 